\title{
LA ETNOGRAFIA EDUCATIVA COMO ALTERNATIVA PARA EL PROFES OR INVESTIGADOR DE EDUCACIÓN FÍSICA. ANÁLISIS DE UNA EXPERIENCIA PRÁCTICA
}

\section{Autor}

\section{RESUMEN}

ALFREDO MARTÍN PEÑALVER

PROFESOR FUNCIONARIO DEEDUCACIÓNFÍSICA

I.E.S. «RIBERA DEL BULLAQUE». PORZUNA. CIUDAD REAL (ESPAÑA)

El profesorado de Educación Física debe ser docente en su tiempo y esto implica, hoy en día, que el profesor sea investigador de su propia práctica.

En este sentido se presenta una experiencia práctica llevada a cabo en el I.E.S. "Ribera del Bullaque" de Porzuna (Ciudad Real), dónde un docente-investigador externo, utiliza la Etnografía Educativa para conocer la motricidad de los alumnos/as en los recreos.

A través del presente estudio, hemos obtenido datos interesantes que nos pueden ayudar en la labor docente de todas las áreas curriculares y de forma muy específica en la Educación Física. El recreo es un espacio de gran riqueza, es el parámetro espacio-temporal propio del alumno dónde se observan sus conductas verdaderas sin ningún tipo de máscara.

\section{PALABRAS CLAVES}

Profesor-investigador, etnografía educativa, investigación cualitativa.

\section{Introducción}

En Etnografía Educativa, la determinación del tema de estudio puede estar condicionada por una serie de factores como por ejemplo: la propia historia personal, ciertos problemas puntuales en la dinámica del proceso de enseñanza-aprendizaje o simplemente por el propio interés profesional. Goetz y LeCompte $(1888,64)$ señalan que "las preguntas que formulan los investigadores están influidas, explícita o implícitamente, por sus experiencias personales y orientaciones filosóficas, que modelan sus intereses y su forma de pensar".

En este sentido, Locke (citado en Fernández-Balboa, J.M. $(1998,3)$ afirma "que la investigación cualitativa, a diferencia de la cuantitativa, no parte de una hipótesis sino que surge a raíz de una pregunta o problema que no es cuantificable". Es decir, un proyecto de investigación etnográfico, a diferencia de uno cuantitativo, no parte de un planteamiento del problema sumamente preciso, sino de una situación problemática reflejada en grandes interrogantes que guían la iniciación del contacto con los actores sociales. En el campo de la Educación se plantean recurrentemente temas que son necesarios abordar en la perspectiva de lograr una comprensión.

En nuestro caso, el presente artículo forma parte de una experiencia de investigación etnográfica llevada a cabo en el I.E.S. "Ribera del Bullaque" de Porzuna (Ciudad Real), durante el curso académico 2001/2002 con el objetivo de observar, profundizar y comprender la motricidad de los alumnos/as en los tiempos de recreo. Dicha experiencia cubrió la fase de docencia del programa de doctorado "Educación Física Escolar"del departamento de Expresión Corporal y Musical de la Facultad de Educación de la Universidad Complutense de Madrid.

\section{Marco teórico y objetivos de la investigación}

Con la implantación de la Ley Orgánica de Ordenación General del Sistema Educativo (L.O.G.S.E.) de 3 de Octubre de 1990, muchas son las cosas que se han modificado, entre ellas el perfil del educador. Al docente se le exige que sea competente, que tome decisiones, que trabaje en equipo y que sea reflexivo y crítico.

En relación a esta última característica, lo que se busca es un profesional que esté continuamente aprendiendo de su trabajo, de su práctica profesional. La capa- 
cidad de investigar sobre la práctica nos permite innovar en la enseñanza y lo que es más importante ser germen de una actualización continua de nuestra actuación como docentes. Además, la investigación en el área de Educación Física, nos puede servir para conseguir el logrado reconocimiento y prestigio académico y profesional.

Tenemos que ser docentes de nuestro tiempo y esto implica ser profesor investigador de nuestra práctica. El concepto de profesor investigador tiene su origen en los trabajos de Stenhouse (1984) basado en la necesidad de que éstos construyan su propio proyecto educativo, investigando sobre su práctica.

Desde esta perspectiva, el docente tiene a su alcance para poder investigar la etnografía educativa, en tanto que método cualitativo. Según Goetz y LeCompte $(1988,41)$ "el objeto de la etnografía educativa es aportar valiosos datos descriptivos de los contextos, actividades y creencias de los participantes en los escenarios educativos". Según Pérez $(1989,126)$ "la metodología etnográfica y los estudios clínicos son particularmente apropiados para la investigación descriptiva del aula y para captar los significados que subyacen al flujo natural de los acontecimientos". Camargo $(1995,54)$ afirma que la "etnografía se plantea como una posibilidad de internarse en la dinámica de una realidad social concreta, ya sea de instituciones, grupos, individuos o programas, de sumergirse en ella para vivir su cultura, apropiarla, entenderla y comprenderla. La intención explícita es profundizar sobre esa realidad, ir más allá de 10 que a la superficie es capaz de emerger".

Así pues, El etnógrafo tiene que sumergirse en la cultura y en la vida cotidiana del aula, pero al mismo tiempo tiene que mantener un criterio más o menos objetivo para discernir, observar y analizar todo lo que observa. Goetz y LeCompte (en Sierra Zamorano, 2001, 2) afirma que "la etnografía es un método de investigación que emplean los científicos sociales de orientación fenomenológica, holística y naturalista: fenomenológica porque parte de lo intuitivo, de lo subjetivo, de 10 vivencial; holistica porque trata de percibir y comprender los fenómenos en su totalidad y en las interrelaciones que se establecen con el medio ambiente; y naturalista porque realiza sus investigaciones en el propio ecosistema donde se producen los acontecimientos". De igual forma Rockwell, (en Candela $\left.M^{a} A, 1990,14\right)$ señala que con la etnografía "se pretende reconstruir los aspectos no ducumentados, rescatar lo cotidiano, 10 inconsciente, 10 oculto de la realidad escolar en una construcción de carácter cualitativo y descriptivo del trabajo de campo"

Pero el hecho de investigar por parte del profesoretnógrafo no es suficiente, pues debe respetar algunos aspectos éticos ligados al proceso de investigación. En el planteamiento del problema debemos tener muy presentes ciertos derechos de las personas observadas, tales como la privacidad, la libertad, el anonimato, la confidencialidad, la seguridad. Posteriormente, en el análisis de los datos y en la elaboración de las conclusiones, el investigador debe ser honesto y no debe manipular, inventar o falsificar los resultados aunque los datos nos respondan a las expectativas iniciales o aunque contradigan los valores o creencias del investigador.

Teniendo como referencia estos aspectos los objetivos que nos hemos planteado en la presente investigación se pueden concretar en las siguientes preguntas: ¿qué actividades físicas realizan los alumnos/as en el tiempo de "recreo"? ¿cuál es su variedad?, ¿es excesiva ó es escasa? ¿qué alumnos/as son los que la realizan?, etc.

Los profesores de Educación Física somos "docentes de patio". Es en este lugar donde pasamos buena parte de nuestra de nuestra vida profesional y, consecuentemente, es el espacio social que presuntamente más conocemos de la escuela. Sin embargo este lugar puede ser un espacio desconocido por los docentes, incluyéndose también el profesor de Educación Física.

Los docentes tienen en mente que el "recreo" es el lugar de esparcimiento para los alumnos, dónde pueden liberar las tensiones acumuladas a través de actividades lúdicas, deportivas y recreativas. Sin embargo, debemos mirar de forma más amplia y no "por el agujero de la cerradura" porque las cosas no son como queremos que sean y tampoco son como eran.

Debemos pararnos con renovado asombro en este lugar porque se descubrirán hechos interesantes que nos pueden ayudar en la labor docente de todas las áreas curriculares y de forma muy concreta en la Educación Física. De Certeau (en Pavía, V. 2000,1) afirma que el patio es "un espacio físico y simbólico con significados especiales en la historia de cada jugador, de cada escuela, de cada comunidad'. Es un espacio poco valorado pero de gran riqueza porque significa el espacio de ruptura entre el adentro y el afuera de la escuela, entre lo obligado y lo libre, entre el alumno y el jugador, entre lo correcto y lo apetecible, etc.

El recreo es el parámetro espacio/temporal propio del alumno dónde se observan sus conductas verdaderas sin ningún tipo de máscara o condicionante cultural. Son conductas o comportamientos motrices, verbales, actitudinales, sociales, etc...; que muestran a los alumnos/as tal y como son. Consecuentemente, si queremos conocer a los alumnos en profundidad hay que estudiarlos en los recreos.

\section{Metodología}

Para estudiar la "motricidad en los recreos" hemos utilizado tres técnicas de recogidas de datos: los registros, los informes y las fotografías. El registro consiste en intentar plasmar la realidad tal y como ocurre en una planilla de observación, mientras que el informe busca la explicación del registro. En todo momento se han utilizado las tres técnicas sin que los alumnos/as lo supieran para no condicionar la propia investigación.

En este sentido la Etnografía Educativa se ha llevado a cabo únicamente con un observador-investigador externo, alejándose, pues, de los planteamientos de Elliot, MacDonald-Ros, Kemmis, Mac Taggart, etc., los cuales planteaban la etnografía educativa con observación participante, dónde el investigador interno y el profesor eran la mismas personas.

La fase práctica de investigación trascurrió desde el 11 de Diciembre de 2001 al 21 de Febrero de 2002. El tiempo de observación se limitó a los recreos de los martes y de los jueves (10:15 -10:30 y 12:20-12:35).

El escenario de investigación ha sido el propio patio del IES "Ribera del Bullaque". Para facilitar la observa- 
ción lo he dividido en zonas: pista polideportiva I, pista polideportiva II, zona próxima al gimnasio, zona de aparcamiento y gravilla y zona interior.

La investigación se centró en todos los alumnos/as del "IES Ribera del Bullaque" de Porzuna (Ciudad Real), el cual aglutina alumnos de diferentes municipios, aldeas, pedanías y núcleos rurales. (Porzuna, Piedrabuena, El Robledo, Las Peralosas, Los Baños del Robledillo, Santa Quiteria, Arroba de los Montes, El Robledillo, El Torno, El Trincheto, etc).

\section{Desarrollo de la investigación}

Un proyecto de investigación etnográfico se entiende como la posibilidad de comprender la realidad social a investigar. Para ello, y como hemos señalado anteriormente, utilizamos tres técnicas de recogidas de datos: los registros y los informes y de forma eventual las fotografías.

A priori parecía sencillo pero, cuándo observamos por primera vez, afloraron infinidad de preguntas como por ejemplo: ¿qué es lo que teníamos que observar en el recreo si los chicos lo que menos hacían era actividad física? ¿teníamos que observar por flujos de comportamiento? ¿qué lugar debíamos elegir para observar?, ¿en qué aspectos debíamos centrar la observación? ¿qué era lo prioritario de la observación?, ¿teníamos que observar la motricidad emergente en espacios como la cafetería y los pasillos?..

Para solucionar estas dudas recurrimos a la ayuda de un experto: Miguel Ángel Sierra Zamorano, profesor del curso "Etnografía Educativa" perteneciente al programa de doctorado: "Educación Física Escolar" del departamento de Expresión Corporal y Musical de la Facultad de Educación de la Universidad Complutense de Madrid.
En la reorientación del trabajo de campo, lo primero fue definir lo que queríamos observar para luego emplear un filtro (una hoja de observación) dónde se dejaba pasar la información útil y se rechazaba la redundante. Por lo tanto, el punto de partida fueron los contenidos generales del área de Educación Física en la Educación Secundaría Obligatoria especificados en el Real Decreto de 6 de Septiembre de 1990 por el que se establece el currículo en dicha etapa. (VER CUADRO 1).

Se añadió un cuadrante nuevo de observación que no corresponde con ningún bloque de contenido de Secundaria pero que tiene continuidad con el desarrollo motor de los chicos de la Educación Primaría. Nos estamos refiriendo al bloque de Educación Física de Base en el que se incluyen el esquema corporal, percepción espacial y las habilidades básicas (marcha, carrera salto, transporte, lanzamiento y recepción).

Si analizamos de forma pormenorizada el cuadrante de observación son muchas las preguntas que nos podemos plantear. Por ejemplo: ¿si un chico practica deporte hay que marcar en la planilla de observación el trabajo de coordinación, equilibrio o esquema corporal...? cualquier movimiento implica expresión-comunicación, ¿debemos reflejarlo?, ¿si un alumno juega al baloncesto significa que trabaja la percepción espacial o ciertas cualidades de la condición física?, etc.

Estas preguntas responden a que el movimiento, entendido como un todo, se puede desgranar en su aspecto cualitativo y cuantitativo: en el desarrollo de cualquier deporte de equipo, en tanto que actividad principal de los recreos, observamos una relación con el resto de alumnos y con el objeto de juego, todo ello inmerso en un espacio y un tiempo determinado. El practicar estos deportes supone tomar una conciencia del propio cuerpo, lo que a su vez implica conocer su cuerpo, controlar la pos-

\section{CUADRO 1}

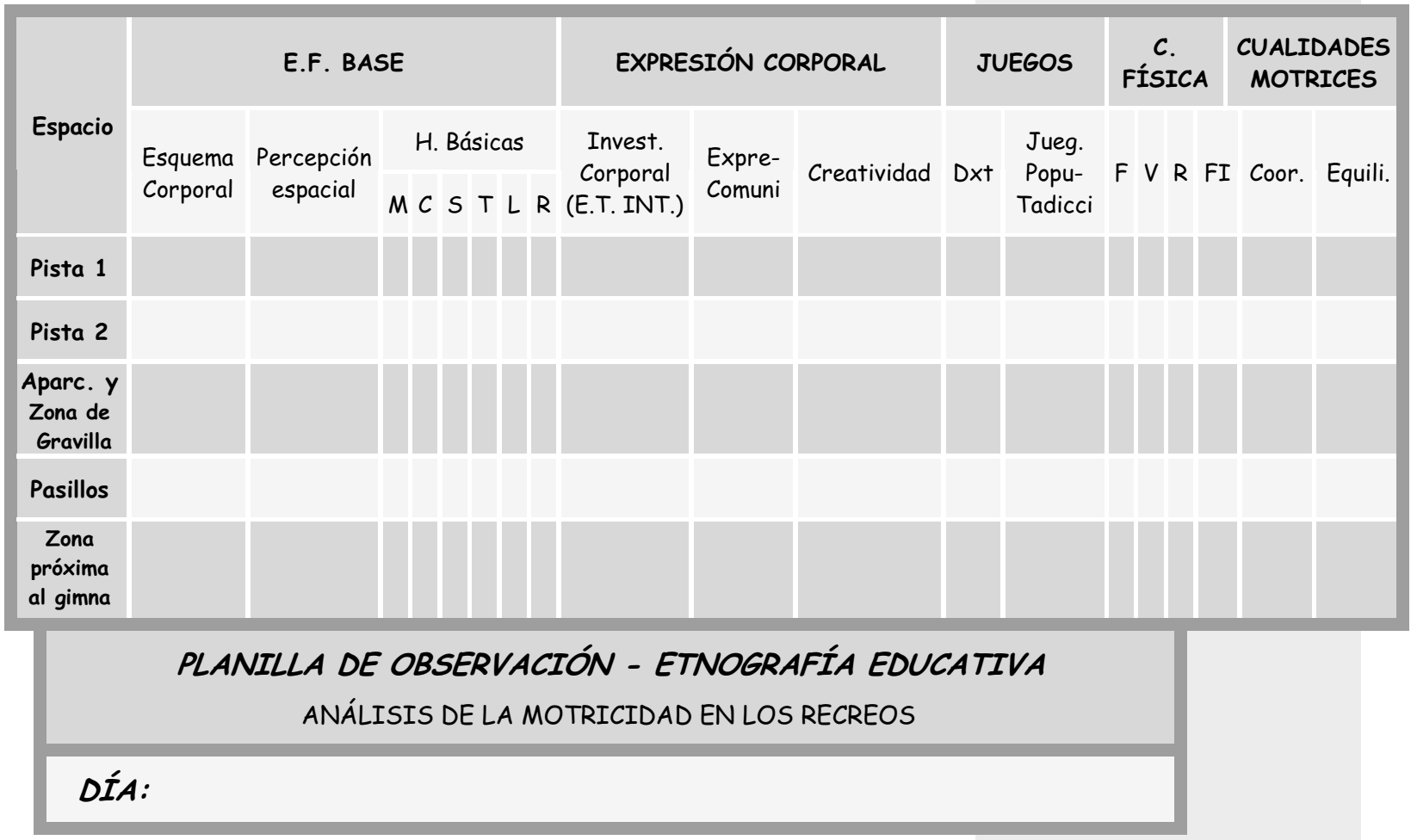


tura tanto estática como dinámicamente, afirmar la lateralidad, etc... En definitiva practicar un deporte de asociación significa tener una buena concepción del esquema corporal, una buena coordinación y una buena percepción espacial.

Todo este análisis es desde un punto de vista cualitativo, sin embargo a nivel cuantitativo en un deporte colectivo también se pueden trabajar ciertas cualidades de la condición física como, por ejemplo, la fuerza, la velocidad, la flexibilidad y la resistencia anaeróbica.

Por consiguiente, de estas ideas se deducen que, si los chicos en el recreo jugaban a un determinado deporte, en nuestra observación teníamos que reflejar lo más evidente sin profundizar si se estaba también trabajando la coordinación, la fuerza, el equilibrio, el esquema corporal, etc...

Dichos aspectos se señalaron única y exclusivamente cuando no sean aspectos secundarios a una manifestación motriz, es decir cuando se evidenciaron de forma muy clara y objetiva.

El papel del análisis de la información cualitativa no es un paso final en el proceso. Es un ejercicio permanente y creativo para organizar y clasificar la información, para ir tejiendo ciertos ejes que vayan configurando nexos del fenómeno estudiado. No es el análisis estadístico convencional de la investigación cuantitativa.

Observar la actividad del recreo se ha convertido en una experiencia atrapante. Describir e interpretar las informaciones no ha sido fácil. En más de una ocasión hemos chocado contra mi propia dificultad de poner en los registros lo que he observado. Los datos son fruto del análisis de los registros y de los informes:

1) El fútbol y el baloncesto son el deporte rey en el recreo; convirtiéndose a su vez en la actividad física principal.

2) El postulado anterior justifica que no existe variedad en las actividades deportivas.

3) En general la actividad física en los recreos podemos catalogarla como mínima.

4) Mayoritariamente los alumnos que practican actividad física en los recreos son los de $1^{\circ}, 2^{\circ}$ de la ESO y en un porcentaje muy reducido los de $4^{\circ}$ de la ESO. Los alumnos de $1^{\circ}$ y $2^{\circ}$ de la ESO juegan al fútbol y los de $4^{\circ}$ de la ESO al baloncesto. (VER FOTO 1).

5) Se han puesto de moda ciertas actividades condicionadas por las prácticas de las clases de Educación Física: malabares y equilibrio invertido.

6) Los alumnos que tienen fidelidad por el deporte lo practican siempre que pueden e incluso en condiciones climatológicas adversas y en combinación de otras actividades; como es por ejemplo: jugar al fútbol y comerse el bocadillo. (VER FOTO 2).

7) Las chicas no practican ninguna actividad deportiva en el recreo y la actividad física que realizan es eventual y mínima en relación a los chicos. Por ejemplo, realizan movimientos expresivos subidas en el banco, buscan el equilibrio corporal al saltar de banco a banco y a lo largo del bordillo.

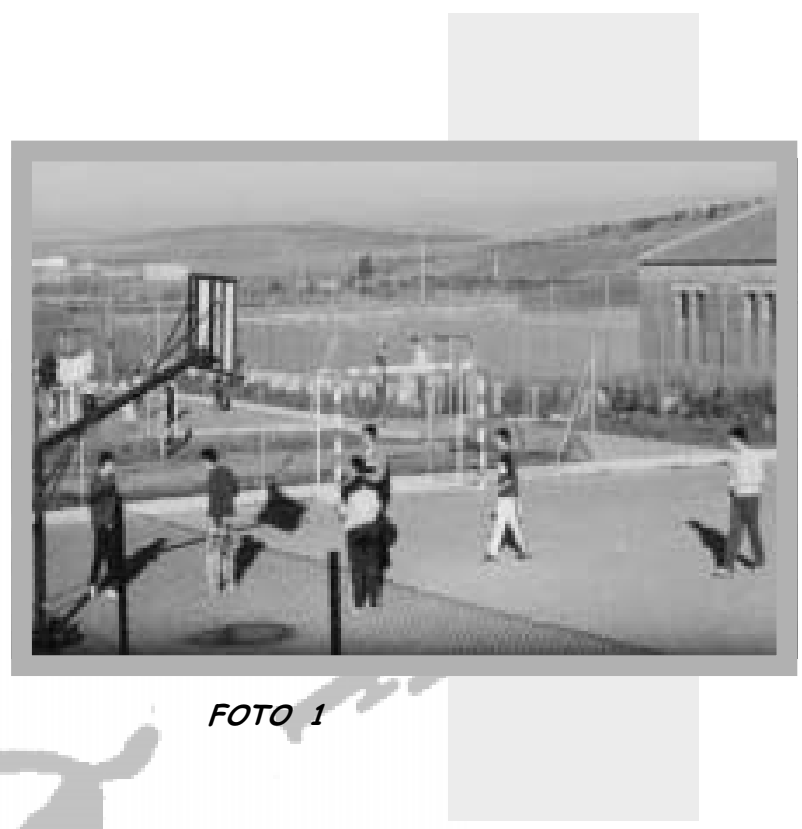

8) Los chicos y las chicas no se relacionan en las actividades físicas de los recreos, pero si en otras actividades como las coloquiales o en las que podríamos catalogar como semiprohibidas (fumar).

9) Los alumnos/as que no realizan actividad física en los recreos se dedican mayoritariamente a ingerir alimentos al tiempo que establecen ciertas conversaciones grupales.

10) Dentro del contenido de Educación Física de Base el que más se ha trabajado ha sido las habilidades básicas y dentro de éste la marcha, la carrera, los lanzamientos y los balanceos.

11) En relación al resto de bloques de contenido los movimientos en general son poco creativos, no practican juegos populares y tradicionales, de forma específica trabajan el equilibrio y las cualidades de la condición física que más trabajan de forma aislada es la fuerza y la velocidad.

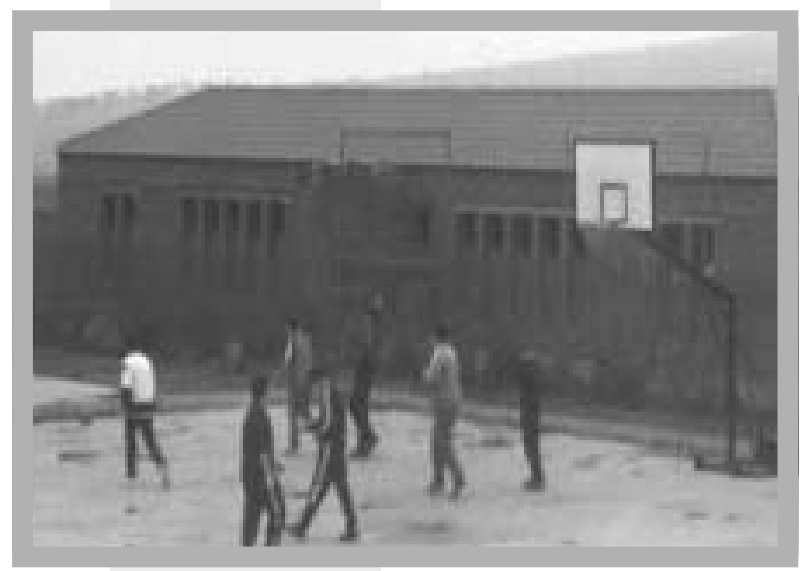

FOTO 2 


\section{Conclusiones}

Una vez que han sido plasmados los resultados debemos formular las conclusiones. En este sentido podemos decir que:

En la población estudiada las especialidades de fútbol y baloncesto presentan unos niveles de práctica muy altos, pero siempre por los mismos alumnos. Esto significa que la práctica de actividad deportiva es mínima si lo extrapolamos al total de alumnos del instituto. Si generalmente son 25-30 chicos los que juegan a estas actividades y en el instituto hay unos 550 alumnos, podemos decir que los alumnos que realizan actividad física de forma intencionada son el 5,5\%.

Esta práctica deportiva en los recreos es inversamente proporcional a la edad y su finalidad es catártica, es decir busca la liberación y compensación de las actividades propiamente del aula. Para estos alumnos el recreo es sinónimo de "pista polideportiva".

Como hemos podido deducir de nuestras observaciones, cualquier espacio o material tiene unas características que desencadenan acciones concretas. Por ejemplo: en el césped es muy probable que alguien se tire, se tumbe o haga una voltereta; en un arenero pueden aflorar ciertos juegos populares y tradicionales. Con el material ocurre lo mismo, una pelota incita a lanzarla, una cuerda a saltar, una rueda a desplazarla, etc. Esto es lo que ha ocurrido en los recreos cuando los chicos que normalmente juegan al fútbol se han encontrado sin balón. Han utilizado el material circulante como, bolsas de plástico, botes de refrescos, lastres de la red de voleibol, elementos físicos como las piedras. Por lo tanto, como facilitadores del aprendizaje podemos condicionar las actividades que realizan los chicos en los recreos introduciendo nuevos materiales en este entorno.

Otro aspecto condicional e interesante en la práctica de actividad física en los recreos ha sido el propio contenido desarrollado en las clases de Educación Física, pues hemos podido observar que los chicos practicaban ciertas actividades llevadas a cabo en clase. Por esta razón en los recreos podemos de jar material a los alumnos así como la utilización de ciertas instalaciones (polideportivo). Desde esta perspectiva vemos interesante que desde la asignatura de Educación Física se oferten ciertas actividades que cultural o socialmente están asignadas al género femenino para instaurarlas así en los recreos y por ende, fomentar la práctica de actividad física en las chicas.

De igual forma y debido a esta influencia, como profesores debemos fomentar desde la Educación Física actividades dónde se trabajen ciertos elementos no visualizados en la motricidad de los recreos, como por ejemplo: actividades de expresión corporal y juegos populares y tradicionales.

Además, la práctica en el recreo de actividades que se han desarrollado en las clases de Educación Física, pueden servir de indicador de autoevaluación para el profesor, en este caso como autoevaluación positiva.

Los alumnos que practican alguna actividad física en los recreos se mantienen al margen de ciertas actividades de riesgo como la inactividad y el hábito de fumar. Esto significa, que las actividades físicas que realizan los alumnos en los recreos condicionan y refuerzan su desarrollo global (cognitivo, afectivo, social y motriz) y su autorrealización personal. A través de estas actividades los alumnos se integran en el espacio escolar, en el barrio, en su pueblo, etc.

Es un elemento vital para la exploración corporal, las relaciones con los demás y el disfrute creativo del ocio. Además al practicar estas actividades físicas el alumno/a está condicionado a desarrollar ciertas actitudes y hábitos de tipo cooperativo y social basados en la solidaridad, la tolerancia, el respeto y la aceptación de las normas de convivencia.

El análisis sociocultural de una etnografía no es patrimonio exclusivo del investigador. Significa que los propios actores de la investigación tienen mucho que decir sobre su cultura. En este sentido, he utilizado un informador clave para que me dijera como organizaban los partidos de fútbol. Este chico me dijo que se distribuían por pueblos, tomando como referencia un agente geográfico: el rio Bullaque..

En definitiva, los recreos son fundamentales en la actividad diaria del alumno en el centro porque, como hemos visto, se observan las conductas reales de los alumnos/as, es el lugar para conocerlos mejor y para poder llevar a cabo una adecuada intervención educativa, formativa, preventiva y reeducativa.

\section{Bibliografía}

Arnold, P.J. (1991). Educación Física, movimiento y currículum. Madrid: Morata.

Camargo, M. (1995). Etnografía de la Adolescencia. Proyecto, adolescencia y escuela. Tomo I. Santafé de Bogotá: T.M. Editores.

Candela, M.A. (1990). La investigación etnográfica en el aula: el razonamiento de los alumnos en una clase de ciencias naturales en la escuela primaria. Revista de investigación en la escuela, 11, 13-23.

Fernández, Balboa, J.M.(1998). La ética en la investigación cualitativa en la Educación Física: teorías, rigor empírico y aspectos éticos en el proceso de la investigación. Revista electrónica ASKESIS, 3, 1-9.

Goetz, J.P. y Lecompte, M.D. (1988). Etnografía y diseño cualitativo en investigación educativa. Madrid: Morata.

Ley Orgánica de Ordenación General del Sistema Educativo de 3 de Octubre de 1990.

Pavía, V. (2000). Investigación y juego, reflexiones desde una práctica. Revista digital de Educación Física, 18,1-4.

Pérez, G. (1994). Investigación cualitativa. Retos e interrogantes. Madrid: La Muralla.

Real Decreto de 6 de Septiembre de 1991 por el que se establece el currículo de la Educación Secundaría Obligatoria.

Sierra, Zamorano, M.A. La Etnografía Educativa con observación participante como medio de formación permanente del profesorado de Educación Física. Revista electrónica ASKESIS, 11, 1-12.

Woods, P. (1989). La escuela por dentro. La etnografía en la investigación educativa. Madrid: Paidós. 